\title{
Review of Context Aware Access Control Approaches on Web Data
}

\author{
Rajni Baghla \\ Research Scholar, Punjabi University Regional \\ Centre for Information Technology and \\ Management, Mohali, Punjab, India
}

\author{
Rekha Bhatia \\ Assistant Professor, Punjabi University Regional \\ Centre for Information Technology and \\ Management, Mohali, Punjab, India
}

\begin{abstract}
The 21 st century is the age of netizens. Internet has become a source of knowledge and platform for social media. Social networking sites have gained huge momentum among all age groups and the popularity has attracted users to devote hours on social networking and contributing to a huge repository of information at every minute. Privacy of this information becomes a critical function of the database. Since personal information of the user is subject to cyber crimes therefore, database level privacy is a fundamental requirement to protect the data. The rapid growth of web on the basis of applications and information system have farther expanded the risk exposure of databases and therefore, nowadays data protection is more important than previous. It is more significant to safeguard data not only from external intruders but also internal intruders. In this paper the various access control scheme and its models to attain the confidentiality, integrity and possibility of objectives of database security in the organization are discussed.
\end{abstract}

\section{Keywords}

Privacy, access control, web, Xml, ontology

\section{INTRODUCTION}

With the growing demand of cloud computing, data can be stored in the cloud and shared between recognized parties. Although, sharing of data enlarge the complication of key distribution. To manage the system in a best way it is required to store data in different types of clouds such s public and private. Data sharing application is specifically designed to deliver protective interaction between larger scalable systems.

Nowadays, in organization permission is required to access data for several purposes monitored by complex policies. These policies are expressed in various forms such as natural language, XML-based formats or firewall code. Because of their size, distribution and complexity, these policies contain errors which result in security susceptibility.

Not long ago, cryptographic schemes have been developed to support access control on the cloud. One of the most revealing mechanism is attribute based encryption which imposed attribute on the basis of access control strategies. The practicality of using these strategies to tackle representation access control problem. To establish and using cryptography is to support flexible access control is essential.

Generally, cryptography delivered protected communications over the unsecured channels. With the use of cryptography, protected data can be attained for the reason that encryption can deliver a manageable form of access control. More complicated situations have been considered where users do not trust everybody with the similar information.
The comprehensive use of Web services, systems has become more linked and united. To protect the information incprporate in these systems there is a need of suitable security and privacy support. Consequently, a large amount of attention is needed to access control policy language for web services which contain diverse environment related to web. The main aim of these languages is to be adjustable and expandable.

Access control is a crucial part of information security. It main motive is to preserve the confidentiality, integrity and availability by restricting access to secured resources and information via reorganization. Depending on specific designs of computer systems, various access control models and systems have been popularized.

With the use of logical formulas to allow or deny access is beneficial because of the following reasons because it is simple and easy, creation of new rule for allowing the access is simple. Second is the flexibility, even in complex policies rules are easy to specify, no limit on the attribution use in a rule or how complex the language to specify the rule.

While many perspectives have been proposed they still has some drawbacks. Firstly, the flexibility and operability and secondly, in policies and access control mechanism.

\section{LITERATURE REVIEW}

Vladimir Koloski et al have presented a formalization of XACML using descriptive logics (DL), which are decidable fragment of first order logic. This interpretation permits covering a more demonstrative subset of XACML than propositional logic on the basis of investigation tools and moreover, they deliver a new investigation service (policy redundancy). The authors deliver empirical assessment of a policy investigation tool that was administered on top of open source DL reasoner Pellet. In this paper, the authors addressed this problem for XACML by proposing clarification on the basis of a decision fragment of FOL. They were able to deliver an identical suite of investigation services alike propositional logic based tools, although adding extra expressiveness by outline sdescribeubjects, actions and resources using ontologies. Furthermore, also showed how common policy constraints, such as role cardinality, separation of duty and role hierarchies can be easily captures by these logics. Finally they evaluated through empirical illustration that off the shelf DL reasoners are practical as XACML investigation tools [1].

William C. Garrison et al have showed that the cryptographic evaluation of dynamic access controls on untrusted platforms acquire computational costs that are likely excessive inpractice. Particularly, they established lightweight constructions for accomplishing role on the basis of access controls over cloud hosted files with the use of identity based and traditional public key cryptography. This is done under a hazard model as close as possible. It has been proved that the 
correctness of constructions and leverage real world RBAC datasets and recent methods established by access control community to experimentally investigate via simulation. They describe a number of bottlenecks and beneficial areas for farther work that will lead to more natural and systematic constructions for the cryptographic accomplish of dynamic access controls. In this paper, they deliver proof that given the existing state of art, in situations including even a reduced amount of policy dynamism, the cryptographic implementation of access controls is likely to conduct prohibitive costs. [2].

Adam Bates,Kevin R.B. Butler have proposed a unique approach to policy on the basis provenance pruning leverage the confinement properties provided by Mandatory Access Control (MAC) systems in order to describe sub domains of system activity for which to collect provenance. They evaluate that adding a policy component to the Hi-Fi provenance monitor can minimized overhead by as much as $82 \%$. According to them, they established first practical policy - based provenance maintained to be proposed the sketch design of a policy based provenance collection startegy and suggested a means of validating the completeness property through resolve the provenance log with MAC information flows [3].

Riaz Ahmed Shaikh et al have presented a unique technique for identifying divergence and shortcoming in access control guidelines with the help of a data investigation tools well known in data mining. The suggested technique composed of three phases: (i) parsing on policy dataset which involves ordering of attributes and normalization of Boolean expressions. (ii) Produced decision trees with the help of their suggested anomaly identification algorithm which is a modification of C4.5 algorithm. (iii) Executed the suggested deviation apprehension algorithm on resulting decision trees. This explanation support to prohobit, identify and exact human errors and expand the capacity for tackling larger and more difficult access control policy sets. With the use of this result, access management security will become more tough and will attain beneficial diagnostic features [4].

Xinfeng Ye have recommended an access control strategy which is a combination of Discretionary access control and cryptographic methods to safeguard users' data and applications. The suggested mechanism permits the users to minister their access acceptance to other users easily. This strategy uses cryptographic methods to vague the access paradigm and users endorsement to establish the privacy of cloud users. This mechanism is more adjustable, systematic and easy to use as compared to other strategy. This mechanism showed that the time for produce an attribute key is comparative to produce an RSA key and when the size of single attribute key is 256 bits with no more than ten attributes in the endorsement or access control rule, the policy enforcement carries out in less than $35 \mathrm{~ms}$. [5].

Prosunjit Biswas et al have proposed an attribute on the basis of access control (ABAC) model namely LaBAC(Label Based Access Control) which adapts the itemized style for expressing approval protocols. An endorsement on policy in $\mathrm{LaBAC}$ for an action is an identification with the use of these two attributes. It can be examined as a bare reduced ABAC model. They reveal that how to composed the traditional RBAC and LBAC models in LaBAC to interpret its expressiveness. The authors investigate $\mathrm{LaBAC}$ model with other endorsed protocol models. There are some problems that need to be addressed to greater recognize the nature of ABAC like: - (i) are there other alternatives to classify authorization policies [6].

Rekha Bhatia et al have suggested a structure that addresses client's privacy matter in the context of web services environment. This path involves service suppliers saving their privacy guidelines in the form of an system class and service users saving their confidential preferences in the form of a rule particular in the syntactic web rule language. This groundwork delivered computerized reasoning methods for matching the service supplier privacy policies for observance with the client's privacy preferences. This structure base computerized propagation of the list of service supplier who agreed to deliver service. By interpretation SWRL rule on the basis of confidential preferences of requesting user with the isolation domain outlook, one can choose the advanced web service supplier from a host of service delieverer and safeguard the user privacy systematic as well as organized. They examined the future work which will analyze the use of controlled natural language (CNL) [7].

Fatemeh Rezaeibagha have inscribe the security and privacy problems of EHR data sharing with their unique access control scheme which apprehension the summary of access control policy transmission, to deliver safeguard and confidential preventing data sharing between distinct health care enterprises. They established an access control strategy with some cryptographic building blocks and conferred a unique prospect for safe EHR systems for hybrid clouds. A efficient study has been carried out on data sharing in EHR systems to deliver a solution to the security and privacy problems. [8].

\begin{tabular}{|c|c|c|c|c|}
\hline AUTHORS & PAPER TITLE & TECHNIQUE USED & MERITS & DEMERITS \\
\hline $\begin{array}{lr}\text { Vladimir Koloski } \\
\text { et } \\
\text { [1],In Proceedings } \\
\text { of the } 16 \text { th } \\
\text { international } \\
\text { conference } \\
\text { World on } \\
\text { Web (pp. } \\
\text { 686). ACM. }\end{array}$ & $\begin{array}{l}\text { Analyzing Web Access } \\
\text { Control Policies }\end{array}$ & $\begin{array}{l}\text { The Authers presented a } \\
\text { formalization of XACML } \\
\text { using description logics (DL), } \\
\text { which allows to cover a more } \\
\text { expressive subset of XA- } \\
\text { CML than prop-ositional } \\
\text { logic based analysis tools, and } \\
\text { provided a new analysis } \\
\text { service (policy redundancy). }\end{array}$ & $\begin{array}{l}\text { These Policies are not } \\
\text { particularly designed for } \\
\text { enforcement, and even } \\
\text { where they could be used } \\
\text { to optimize enforcement. } \\
\text { Optimization can be done } \\
\text { offline. }\end{array}$ & $\begin{array}{l}\text { These services } \\
\text { can be } \\
\text { computationall } \\
\text { y expensive. }\end{array}$ \\
\hline $\begin{array}{l}\text { William C. } \\
\text { Garrison et al [2], }\end{array}$ & $\begin{array}{l}\text { On the Practicality of } \\
\text { Cryptographically } \\
\text { Enforcing Dynamic } \\
\text { Access Control Policies }\end{array}$ & $\begin{array}{l}\text { The authors developed } \\
\text { constructions for enforcing } \\
\text { role-based access controls } \\
\text { over cloud-hosted files using }\end{array}$ & $\begin{array}{l}\text { This work lead to more } \\
\text { natural and efficient } \\
\text { constructions for the } \\
\text { cryptographic }\end{array}$ & $\begin{array}{l}\text { the techniques } \\
\text { used under this } \\
\text { work are not } \\
\text { compatible }\end{array}$ \\
\hline
\end{tabular}




\begin{tabular}{|c|c|c|c|c|}
\hline & in the Cloud & $\begin{array}{l}\text { identity based and traditional } \\
\text { public-key cryptography } \\
\text { under a threat model. }\end{array}$ & $\begin{array}{l}\text { enforcement of dynamic } \\
\text { access controls. }\end{array}$ & $\begin{array}{l}\text { with hybrid } \\
\text { encryption } \\
\text { which is } \\
\text { necessary from } \\
\text { an efficiency } \\
\text { perspective } \\
\text { under } \\
\text { reasonable } \\
\text { threat models. }\end{array}$ \\
\hline $\begin{array}{lr}\text { Adam Bates, } \\
\text { Kevin R.B. Butler } \\
\text { [3], } \\
\text { USENIX } \\
\text { Workshop } \\
\text { Theory } \\
\begin{array}{l}\text { Practice } \\
\text { Provenance }\end{array} \\
\text { 15). }\end{array}$ & $\begin{array}{lr}\text { Take Only } & \text { What You } \\
\text { Need: } & \text { Leveraging } \\
\text { Mandatory } & \text { Access } \\
\text { Control Policy to } \\
\text { Reduce Provenance } \\
\text { Storage Costs }\end{array}$ & $\begin{array}{l}\text { The authors proposed an } \\
\text { approach on the basis } \\
\text { provenance pruning - } \\
\text { leverage the confinement } \\
\text { properties provided by } \\
\text { Mandatory Access Control } \\
\text { (MAC) systems to describe } \\
\text { sub domains of system } \\
\text { activity. }\end{array}$ & $\begin{array}{l}\text { This is the first practical } \\
\text { policy - based } \\
\text { provenance maintained to } \\
\text { be proposed the sketch } \\
\text { design of a policy based } \\
\text { provenance collection } \\
\text { startegy and validating } \\
\text { the completeness } \\
\text { property through the } \\
\text { provenance log. }\end{array}$ & $\begin{array}{l}\text { This approach } \\
\text { provides } \\
\text { selective } \\
\text { completeness } \\
\text { properties. }\end{array}$ \\
\hline $\begin{array}{l}\text { Riaz Ahmed } \\
\text { Shaikh et al [4], } \\
\text { International } \\
\text { Journal of } \\
\text { Information } \\
\text { Security, 1-23. }\end{array}$ & $\begin{array}{l}\text { A Data Classification } \\
\text { Method for } \\
\text { Inconsistency and } \\
\text { Incompleteness } \\
\text { Detection in Access } \\
\text { Control Policy Sets }\end{array}$ & $\begin{array}{l}\text { The authors proposed a } \\
\text { method which provides } \\
\text { means for handling } \\
\text { incompleteness, continuous } \\
\text { values and complex Boolean } \\
\text { expressions. }\end{array}$ & $\begin{array}{l}\text { The technique used by } \\
\text { these authors is generic, } \\
\text { i.e. independent of any } \\
\text { policy specification } \\
\text { language. By using this } \\
\text { technique, access } \\
\text { management security } \\
\text { products will become } \\
\text { more robust and will gain } \\
\text { useful diagnostics } \\
\text { characteristics. }\end{array}$ & $\begin{array}{l}\text { Loss of assets } \\
\text { and prestige } \\
\text { are some of the } \\
\text { possible } \\
\text { Consequences. }\end{array}$ \\
\hline $\begin{array}{l}\text { Xinfeng Ye [5], } \\
\text { Tsinghua Science } \\
\text { and } \\
\text { Technology, 21(0 } \\
\text { 1), 40-54. }\end{array}$ & $\begin{array}{l}\text { Privacy Preserving and } \\
\text { Delegated Access } \\
\text { Control for Cloud } \\
\text { Applications }\end{array}$ & $\begin{array}{l}\text { The authors have proposed an } \\
\text { access control scheme which } \\
\text { uses a combination of } \\
\text { discretionary access control } \\
\text { and cryptographic techniques } \\
\text { to secure user's data and } \\
\text { applications hosted by cloud } \\
\text { providers. }\end{array}$ & $\begin{array}{l}\text { Compared with existing } \\
\text { schemes, the proposed } \\
\text { scheme is more flexible, } \\
\text { efficient and easy to use. }\end{array}$ & $\begin{array}{l}\text { User's task is } \\
\text { limited to } \\
\text { generating } \\
\text { credential key } \\
\text { and Policy } \\
\text { keys. }\end{array}$ \\
\hline $\begin{array}{l}\text { Prosunjit Biswas } \\
\text { et al [6], In } \\
\text { Proceedings of the } \\
2016 \text { ACM } \\
\text { International } \\
\text { Workshop on } \\
\text { Attribute Based } \\
\text { Access } \\
\text { Control (pp. } \\
\text { 12). ACM. }\end{array}$ & $\begin{array}{lr}\text { Label-Based } & \text { Access } \\
\text { Control: An ABAC } \\
\text { Model } & \text { with } \\
\text { Enumerated } & \\
\text { Authorization Policy }\end{array}$ & $\begin{array}{l}\text { The authors have proposed an } \\
\text { attribute on the basis of } \\
\text { access control model namely } \\
\text { LaBAC(Label Based Access } \\
\text { Control) which adapts the } \\
\text { itemized style for expressing } \\
\text { approval protocols. }\end{array}$ & $\begin{array}{l}\text { They also discussed } \\
\text { logical formula based } \\
\text { authorization policy } \\
\text { which is the more } \\
\text { conventional approach } \\
\text { for designing ABAC } \\
\text { policy which can be very } \\
\text { rich and complex and } \\
\text { capable of expressing } \\
\text { even complicated } \\
\text { business logic. }\end{array}$ & $\begin{array}{l}\text { The cost of } \\
\text { storing } \\
\text { potentially } \\
\text { large number } \\
\text { of enumerated } \\
\text { tuples may be } \\
\text { very high. }\end{array}$ \\
\hline $\begin{array}{l}\text { Rekha Bhatia et al } \\
\text { [7], } \\
\text { In Proceedings of } \\
\text { International } \\
\text { Conference on } \\
\text { ICT for } \\
\text { Sustainable } \\
\text { Development (pp. } \\
\text { 475-484). } \\
\text { Springer } \\
\text { Singapore }\end{array}$ & $\begin{array}{lrr}\text { An Implementation } \\
\text { Model for } & \text { Privacy } \\
\text { Aware } & \text { Access } & \text { Control } \\
\text { in Web } & \text { Services } \\
\text { Environment } & \end{array}$ & $\begin{array}{l}\text { The authors have suggested a } \\
\text { structure that addresses } \\
\text { client's privacy matter in the } \\
\text { context of web services } \\
\text { environment. }\end{array}$ & $\begin{array}{l}\text { This framework provides } \\
\text { automated reasoning } \\
\text { techniques for matching } \\
\text { the service provider's } \\
\text { privacy policies with the } \\
\text { client's privacy } \\
\text { preferences. }\end{array}$ & $\begin{array}{l}\text { the work using } \\
\text { controlled } \\
\text { natural } \\
\text { language } \\
\text { (CNL)-based } \\
\text { interfaces for } \\
\text { specification of } \\
\text { privacy } \\
\text { preferences by } \\
\text { the users in } \\
\text { order to lessen } \\
\text { the burden on } \\
\text { user is yet to be }\end{array}$ \\
\hline
\end{tabular}




\begin{tabular}{|c|c|c|c|c|}
\hline & & & & done. \\
\hline $\begin{array}{l}\text { Fatemeh } \\
\text { Rezaeibagha [8], } \\
\text { International } \\
\text { Journal } \\
\text { Medical } \\
\text { Informatics, 89, } \\
25-31 .\end{array}$ & $\begin{array}{l}\text { Distributed clinical data } \\
\text { sharing via dynamic } \\
\text { access-control policy } \\
\text { transformation }\end{array}$ & $\begin{array}{l}\text { The authors have proposed an } \\
\text { access control strategy with } \\
\text { some cryptographic building } \\
\text { blocks and conferred a unique } \\
\text { prospect for safe EHR } \\
\text { systems for hybrid clouds. } \\
\text { This hierarchical access } \\
\text { structure grants access to } \\
\text { authorized users and limits } \\
\text { access rights to other users in } \\
\text { the public domain. }\end{array}$ & $\begin{array}{l}\text { Using this approach, } \\
\text { Electronic Health Record } \\
\text { can be stored in multiple } \\
\text { clouds. }\end{array}$ & $\begin{array}{l}\text { Data } \\
\text { redundancy } \\
\text { may occur as } \\
\text { they used } \\
\text { multiple clouds } \\
\text { to store user } \\
\text { data. }\end{array}$ \\
\hline
\end{tabular}

The next section 3 is describing the issues and challenges in the context aware access control approaches on web data

\section{ISSUES AND CHALLENGES}

While reviewing various research papers, some issues and challenges in Context Aware Access Control Approaches on Web Data are come into knowledge. These are explained as following:

1. Privacy preserving policy should be related to all authorized documents.

2. Privacy policy should not use much space for saving policy rules.

3. Policy rules should be non ambiguous and clear about query and access control.

4. Policy's rules should be generalized for different databases, without any need to change.

5. If queries are related to each other, then authorization should not be overlapped.

6. There should be different policies for different levels of database.

7. To test the policies, effective queries should be selected so that efficient results should be obtained.

The issues and challenges section has described about the primary issues and challenges associated with the development of the research proposed in this paper. The next section concludes the research gaps of the existing systems.

\section{RESEARCH GAPS}

On the basis of above defined issues and challenges, there are following research gaps:

- Privacy policy's main requirement is database of policy and rules, these rules should be related to each other and inference the protection of database but sometimes rules over lap each other and give ambiguous result.

- Whenever the privacy policies are used on database it should take less time but, sometimes system takes more time while searching the policies in databases.

- The authorization of database preserving is not inferenced from policies, that is why relation between database is the main requirement to reduce the time complexity.

- Database privacy is important but should be authorized in multilevel, but the use of multilevel ontologies is a big challenge.

\section{CONCLUSION}

We have initiated several new research directions. First, in this paper developed the idea that attacker models should be studied and formalized for databases. Rather than being implicit, the relevant models must be made explicit, just like when analyzing security in other domains. In this respect, we presented a concrete attacker model that accounts for relevant features of modern databases, like triggers and views, and attacker inference capabilities.

Second, access control mechanisms must be designed to be secure, and provably so, with respect to the formalized attacker capabilities. This requires research on mechanism design, complemented by a formal operational semantics of databases as a basis for security proofs. We presented such a mechanism, proved that it is secure, and built and evaluated a prototype of it in PostgreSQL. As a future work, we will extend our framework and our PDP to directly support the SQL language.

\section{REFERENCES}

[1] Kolovski, V., Hendler, J., \& Parsia, B. (2007, May). Analyzing web access control policies. In Proceedings of the 16th international conference on World Wide Web (pp. 677-686). ACM.

[2] Garrison III, W. C., Shull, A., Myers, S., \& Lee, A. J. On the Practicality of Cryptographically Enforcing Dynamic Access Control Policies in the Cloud.

[3] Bates, A., Butler, K. R., \& Moyer, T. (2015). Take only what you need: leveraging mandatory access control policy to reduce provenance storage costs. In 7th USENIX Workshop on the Theory and Practice of Provenance (TaPP 15). 
[4] Shaikh, R. A., Adi, K., \& Logrippo, L. (2016). A Data Classification Method for Inconsistency and Incompleteness Detection in Access Control Policy Sets. International Journal of Information Security, 1-23.

[5] Ye, X. (2016). Privacy preserving and delegated access control for cloud applications. Tsinghua Science and Technology, 21(01), 40-54.

[6] Biswas, P., Sandhu, R., \& Krishnan, R. (2016, March). Label-Based Access Control: An ABAC Model with Enumerated Authorization Policy. InProceedings of the
2016 ACM International Workshop on Attribute Based Access Control (pp. 1-12). ACM.

[7] Bhatia, R., \& Singh, M. (2016). An Implementation Model for Privacy Aware Access Control in Web Services Environment. In Proceedings of International Conference on ICT for Sustainable Development (pp. 475-484). Springer Singapore.

[8] Rezaeibagha, F., \& Mu, Y. (2016). Distributed clinical data sharing via dynamic access-control policy transformation. International Journal of Medical Informatics, 89, 25-31. 